\title{
État de l'art et derniers développements en ICP-MS
}

\section{ICP-MS state of the art and last development}

Hugues PAUCOT*

UT2A, Pau

*Auteur à qui adresser la correspondance : Hugues PAUCOT, UT2A,

Hélioparc Pau Pyrénées, 2, Avenue du Président Angot, 64053 Pau Cedex 9, FRANCE Tél : 0559806882 - Fax : 0559806367 - E-mail : hugues.paucot@univ-pau.fr

(Reçu le 23 février 2007 ; accepté après modifications le 9 mars 2007)

\section{RÉSUMÉ}

Introduits dès 1983, les ICP-MS font dorénavant partie intégrante du parc analytique de nombreux laboratoires impliqués dans l'analyse de traces métalliques. Leur vitesse d'exécution, alliée au caractère multiélémentaire de la technique et à une diminution régulière des coûts, les a progressivement conduits à remplacer nombre d'appareils d'absorption atomique électrothermique. De plus, les modifications apportées sur les générations successives d'ICP-MS ont fortement augmenté l'accessibilité de la technique, tout en permettant de résoudre la plupart des interférences affectant initialement ce type d'appareillage. Ainsi, en ce qui concerne les divers types d'interférences, plusieurs technologies ont été développées, comme celle des appareils dits de haute résolution, les multicollecteurs, le temps-de-vol ou les dispositifs de collision/réaction. Ces derniers systèmes équipant $90 \%$ des ICP-MS quadripolaires vendus actuellement dans le monde, il est maintenant possible de faire un bilan de leurs limites. Les ultimes modifications apportées aux ICP-MS sont en fait des adaptations des appareillages, réalisées en fonction des applications spécifiques de l'utilisateur, telles que : types de gaz de collision/réaction installés par défaut sur les appareils, formes des cônes de l'interface et de leurs supports, etc. Enfin, devant la demande croissante en analyses de spéciation, les constructeurs se sont investis dans la fourniture des accessoires indispensables (interface, logiciel...), ainsi que dans l'automatisation du traitement des signaux transitoires.

\section{MOTS-CLÉS}

ICP-MS, cellule de collision/réaction, interface de collision/réaction, haute résolution, multicollecteur, temps de vol, spéciation.

\section{SUMMARY}

Since their introduction in 1983, ICP-MS are henceforth part of the analytical instrumentation of many laboratories involved in trace metals analyses. Their speed, combined to the multielementary property of the technique and to a regular decrease of the cost, has driven them to replace progressively the graphite furnace atomic absorption spectrometers in many laboratories. Modifications brought on the successive generations have greatly increased the technique accessibility, and allowed to solve most of the interferences initially affecting this kind of instrument.

So, regarding the various types of interferences, several technologies have been developed, like these of the high resolution, multicollectors, time-of-flight or collision/reaction systems. Due to the fact that these last are present on $90 \%$ of ICP-MS actually sold worldwide, it is now possible to strike a balance of limits of this kind of systems. The last modifications brought to ICP-MS are in fact apparatus adaptations, realized as a function of user's specific applications, like : collision/reaction gas type installed by default on the instruments, interface cones and supports designs, etc. Finally, due to the increasing demand for speciation analyses, manufacturers have invested in providing indispensable accessories (interface, software...), but also in the automatisation of transient signal treatment.

\section{KEY-WORDS}

ICP-MS, Collision reaction cell, collision reaction interface, high resolution, multicollector, time-of-flight, speciation. 


\section{Introduction}

Introduit dès 1983, les ICP-MS (Inductively Coupled Plasma - Mass Spectrometer) sont aujourd'hui devenus des appareils incontournables pour la plupart des laboratoires spécialisés dans l'analyse de traces métalliques. Sept constructeurs (plus un constructeur spécifique au marché japonais) (tableau I) se partagent un marché mondial de plus de 800 appareils, dont un marché français s'élevant à 36 unités l'an dernier. Même si leur pénétration ne fût que très progressive en France, et plus particulièrement dans le domaine de la biologie clinique (et ce, malgré les travaux précoces du Professeur Allain (1 - 3)), leur vitesse d'exécution, alliée au caractère multiélémentaire de la technique, aux limites de détection et à une diminution régulière des coûts, les a progressivement conduits à remplacer les appareils d'absorption atomique électrothermique dans bon nombre de laboratoires, entraînant un tassement important du marché français de cette technique.

Toutefois, malgré la technologie innovante apportée par ces appareils, et en particulier par les ICP-MS quadripolaires, il n'en demeure pas moins qu'ils restent handicapés par un certain nombre d'interférences, et en particulier, d'interférences spectrales. Plusieurs solutions ont été développées afin de faire face à cette problématique. Nous traiterons successivement ces différentes évolutions.

\section{Les appareils dits de « Haute Résolution » - ICP-HR-MS}

Dès 1989, la mise sur le marché du premier ICP-MS à Haute Résolution (figure 1), a permis de résoudre nombre d'interférences spectrales, telles que ${ }^{40} \mathrm{Ar}^{12} \mathrm{C}$ sur le ${ }^{52} \mathrm{Cr},{ }^{40} \mathrm{Ar}{ }^{35} \mathrm{Cl}$ sur $1^{175} \mathrm{As}$ ou encore ${ }^{40} \mathrm{Ar}^{16} \mathrm{O}$ sur le ${ }^{56} \mathrm{Fe}$, sans toutefois permettre de les résoudre toutes, comme, par exemple, l'interférence isobarique de ${ }^{40} \mathrm{Ar}$ sur $1 \mathrm{e}^{40} \mathrm{Ca}$. Cette technique est basée sur la déflection des ions en fonction de leur masse et de leur énergie par un champ magnétique et un champ électrique. La trajectoire des ions, longue de près de deux mètres, permet d'obtenir un pouvoir de résolution (défini comme étant le rapport entre la masse moyenne de l'analyte et de l'interférent, divisée par la différence de masse entre ces deux ions) de plus de 12000. Comparativement, celle d'un ICP-MS quadripolaire s'établit, au maximum, aux alentours de 800. Même si les derniers perfectionnements réalisés sur ce genre d'instrument ont permis d'en diminuer fortement l'empreinte au sol et le volume, il n'en demeure pas moins qu'ils restent plus volumineux qu'un ICP-MS quadripolaire classique. De plus, le coût

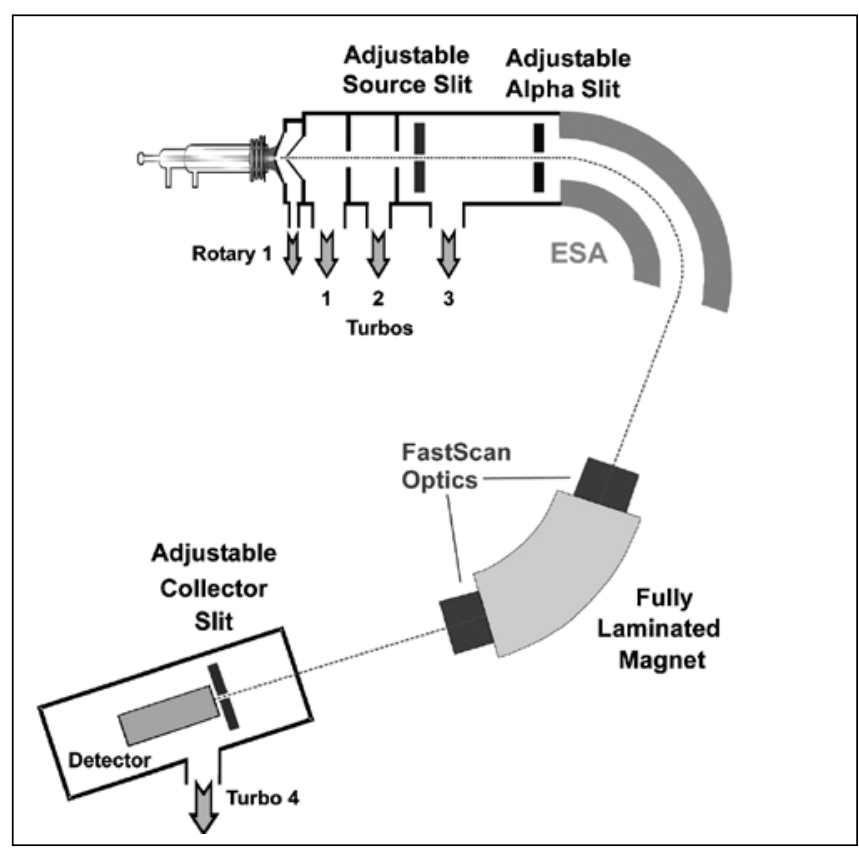

Figure 1 : Schéma d'un ICP-HR-MS (Courtoisie Nu Instruments).

Tableau I : Constructeurs d'ICP-MS distribués en France.

\begin{tabular}{|c|c|c|c|c|c|c|}
\hline \multirow[t]{3}{*}{ Constructeur } & \multicolumn{3}{|c|}{ ICP-Q-MS } & ICP-HR-MS & ICP-MC-MS & ICP-TOF-MS \\
\hline & \multirow{2}{*}{$\begin{array}{c}\text { Sans } \\
\text { dispositif } \\
\text { de } \mathbf{C R} \\
\end{array}$} & \multicolumn{2}{|c|}{ Avec dispositif de CR } & & & \\
\hline & & Cellule & Interface & & & \\
\hline Agilent Technologies & $7500 a$ & $7500 \mathrm{ce} 7500 \mathrm{cs}$ & & & & \\
\hline $\begin{array}{l}\text { GBC Scientific } \\
\text { Équipment (Vinci) }\end{array}$ & & & & & & OptiMass 9500 \\
\hline GV Instruments & & & & & IsoProbe & \\
\hline $\mathrm{Nu}$ Instruments & & & & ATTOM & Plasma 1700, Plasma HR & \\
\hline PerkinElmer & Elan 9000 & Elan DRC-e, DRC II & & & & \\
\hline $\begin{array}{l}\text { Thermo Fisher } \\
\text { Scientific }\end{array}$ & $\mathrm{X}$ Series $^{\mathrm{II}}$ & $\mathrm{X}$ Series ${ }^{\mathrm{II}} \mathrm{CCT}$ & & Element 2 & Neptune & \\
\hline Varian, Inc & 810-MS & & 820-MS & & & \\
\hline
\end{tabular}


de l'investissement en est généralement double voire triple. Si les limites de détection sont généralement aussi bonnes, voire meilleures, que pour les dispositifs quadripolaires, ce type d'appareillage est cependant généralement plus lent en terme d'acquisitions de données du fait du système de séparation des masses. Deux constructeurs se partagent néanmoins ce marché de l'ordre de quelques dizaines d'appareils par an au niveau mondial (tableau I).

\section{Les appareils multicollec- teurs ou ICP-MC-MS}

Outre les ICP-HR-MS, trois constructeurs se partagent celui des multicollecteurs (tableau I) ou ICP-MC-MS. Généralement basés sur le même principe que les appareils de Haute Résolution, ils disposent en outre de la faculté de mesurer simultanément plusieurs masses adjacentes, conservant ainsi une totale simultanéité dans la mesure de plusieurs isotopes du même élément (figure 2). Ce genre d'acquisition est rendu possible par l'utilisation de plusieurs détecteurs (Type cage de Faraday ou multiplicateur d'électrons) disposés côte à côte au sortir du système dispersif. Cette particularité permet en effet de mesurer des rapports isotopiques avec une précision nettement supérieure à celle atteinte par un ICP-MS quadripolaire classique (4), et tout à fait comparable aux spectromètres de masse à ionisation thermique (TIMS). Si les ICP-MC-MS s'avèrent être des instruments extrêmement performants, leur coût de 6 à 8 fois plus élevé que celui d'un ICP-MS quadripolaire les réserve à certaines applications spécifiques telles que la répression des fraudes (5), l'authentification précise des sources de pollution (6) ou encore les études géologiques (7).

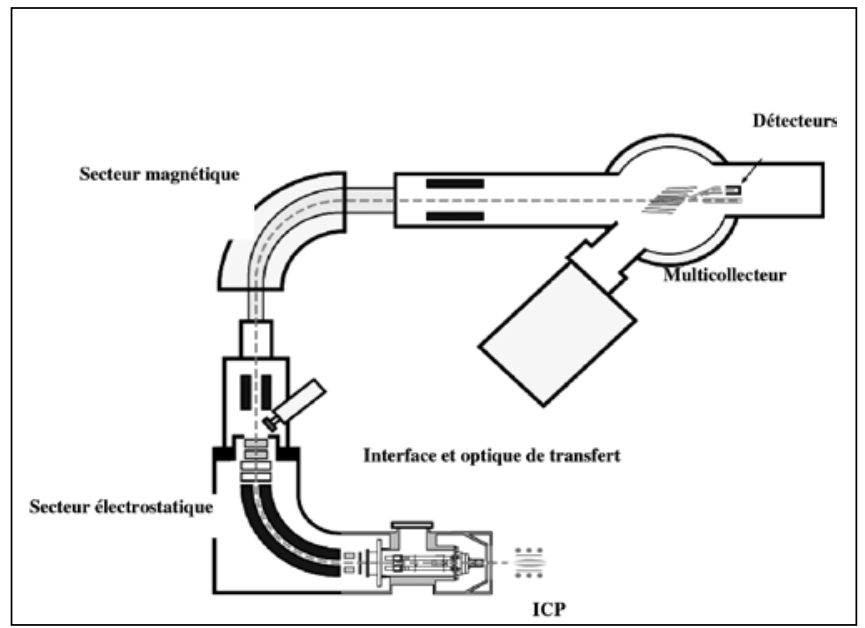

Figure 2 : Schéma d'un ICP-MC-MS (Courtoisie Thermo Fisher Scientific).

\section{L' ICP-MS temps de vol ou ICP-TOF-MS}

A ce jour, il ne subsiste qu'un seul constructeur investit dans la commercialisation d'un ICP-MS à temps de vol (TOF - Time of Flight) (tableau I). Le principe de ce type de machine est basé sur une homogénéisation de l'énergie cinétique des ions dès leur entrée dans le spectromètre et dont il résulte que leur vitesse dans le tube de vol est inversement proportionnelle à leur masse (figure 3) (8). Pour une distance à parcourir donnée, tous les ions arrivent donc au détecteur les uns après les autres. Une électronique rapide permet alors de mesurer successivement l'intensité de chaque masse. L'analyse de l'ensemble des masses du spectre peut alors être réalisée plusieurs milliers de fois par seconde. En outre, si ce type d'instrument a un pouvoir de résolution supérieur à celui des ICP-MS quadripolaires, les limites de détection, quant à elles, sont généralement du même ordre de grandeur. Toutefois l'analyse extrêmement rapide, quasi simultanée, des différentes masses analysées en fait un outil extrêmement intéressant pour la mesure de rapports isotopiques ou pour les analyses de signaux transitoires. Cette propriété peut ainsi être exploitée dans le cadre des analyses de spéciation lors du couplage avec une technique chromatographique (9) ou lors d'introduction spécifique avec système d'ablation laser (10) ou d'injection de flux (11), par exemple.

\section{Les ICP-MS quadripolaires ou ICP-Q-MS}

En ce qui concerne les quadripôles, la principale évolution récente vient de l'adjonction d'un dispositif spécifique destiné à éliminer les interférences spectrales, appelé dispositif de collision/réaction (12). Il a en effet

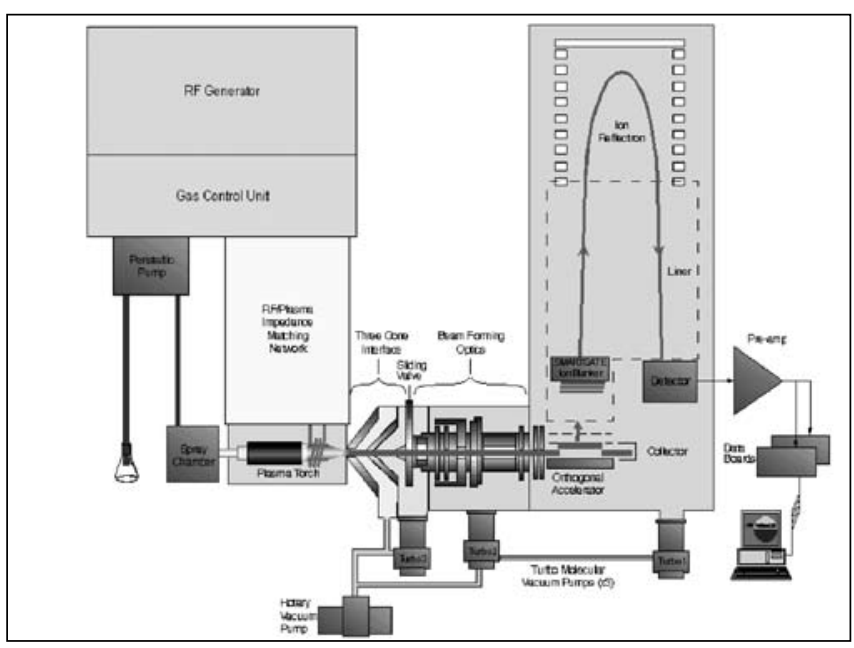

Figure 3 : Schéma d'un ICP-TOF-MS (Courtoisie GBC Scientific Equipment). 
été suggéré, à la fin des années 80 , que ce genre de dispositif pouvait être un moyen efficace, flexible et relativement bon marché de résoudre les interférences spectrales $(13,14)$. Il faudra toutefois attendre 1997, pour voir paraître la première publication décrivant un ICP-MS muni d'une cellule pressurisée incluant un multipôle, installée dans l'optique ionique du système (15). Depuis cette date, plusieurs constructeurs ont introduit sur le marché un appareil de ce type (tableau I). Plus récemment, en 2005, l'introduction de la première interface de collision/réaction (tableau I), a dévoilé une autre possibilité de destruction des interférences spectrales, par l'introduction du gaz directement au niveau des cônes.

Dans tous les cas de figure, le principe fondamental consiste à injecter au niveau du faisceau ionique de l'ICP-MS, un gaz efficace qui interagit avec l'interférent ou l'analyte. Cette injection peut être réalisée soit dans une cellule spécifiquement installée au niveau de l'optique ionique, soit au niveau de l'interface de l'ICPMS. Nous présentons ces deux systèmes dans l'ordre chronologique de leur apparition sur le marché.

Ce genre de dispositif est constitué d'un multipôle enfermé dans une cellule, et pressurisé au moyen d'un gaz ou d'un mélange de gaz qui, selon le cas, est inerte ou potentiellement réactif. Le dispositif est installé au niveau de l'optique ionique de l'appareil. Selon le constructeur, le multipôle peut être un quadripôle, un hexapôle ou un octopôle. Des tensions alternatives sont appliquées à chacun de ces pôles afin d'assurer, au minimum, un guidage des ions vers la sortie de la cellule.

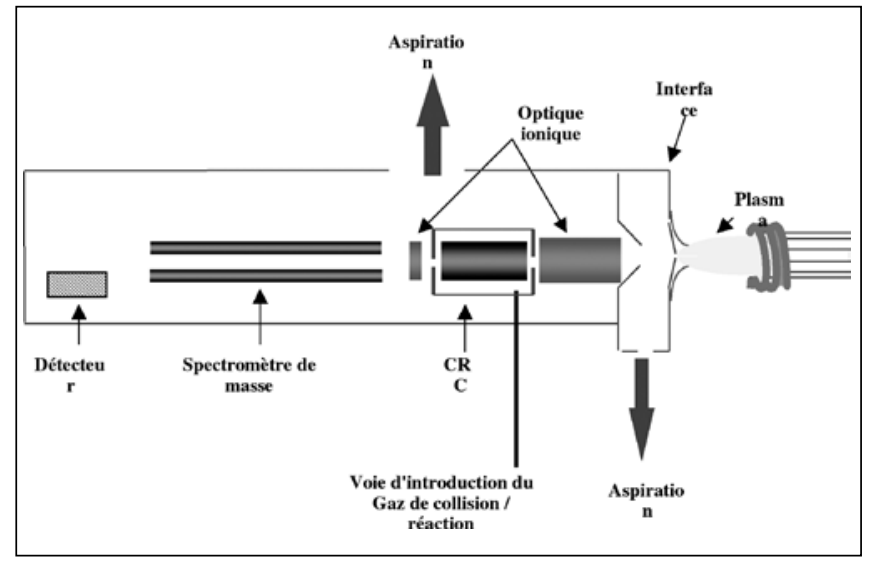

Figure 4 : Schéma d'une cellule de collision/réaction (CRC).

Dans ce cas, l'injection du gaz s'effectue directement au niveau des cônes de l'interface via des petits canaux percés dans l'épaisseur des cônes. Aucune cellule ni multipôle autre que celui du spectromètre de masse, ne sont donc présents sur ce type d'appareillage.

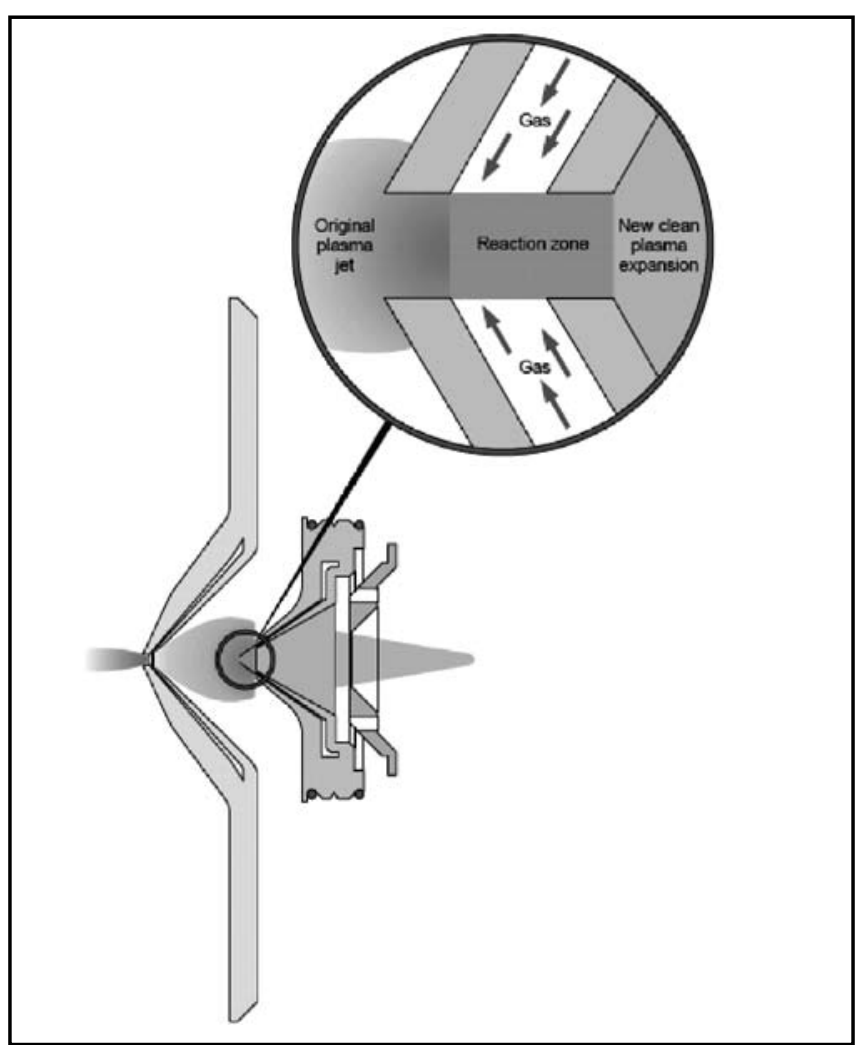

Figure 5 : Schéma d'une interface de collision/réaction (CRI) (Courtoisie Varian, Inc).

Selon le ou les types d'interférences que l'on souhaite résoudre, deux philosophies d'utilisation peuvent être envisagées. La première, de très loin la plus répandue, consiste à modifier ou à détruire l'interférence par un mécanisme collisionnel ou réactionnel. L'autre, plus anecdotique mais surtout utile en cas d'interférences multiples, consistera à modifier la masse de l'analyte par une réaction de condensation, et à analyser le condensat. Le choix du gaz sera déterminé par son efficacité lors des processus collisionnels ou réactionnels, tant d'un point de vue thermodynamique que cinétique. Un gaz de masse atomique faible est généralement utilisé afin de limiter la perte de sensibilité de l'analyte du fait de la dispersion lors des collisions.

\section{Les collisions}

Dans ce cas, le gaz utilisé est généralement non-réactif $(\mathrm{He}, \mathrm{Ar}, \mathrm{Xe} . .$.$) et dissocie l'ion polyatomique interférent$ par simple collision. Ce type d'application reste toutefois limité en ICP-MS, du fait qu'elle nécessite une énergie de liaison des interférents polyatomiques significativement plus faible que l'énergie de collision. Cependant, elle se produit dans une certaine mesure avec plusieurs espèces polyatomiques comme $\mathrm{ArC}^{+}, \mathrm{ArO}^{+}, \mathrm{NaAr}^{+}, \mathrm{MgAr}^{+}$et $\mathrm{CaAr}^{+}$, par exemple.

Toutefois, toute collision entre un ion issu du plasma, et donc pourvu d'une énergie cinétique importante, et l'atome ou la molécule de gaz injecté, s'accompagne 
inéluctablement d'un transfert d'énergie de l'ion vers l'atome ou la molécule de gaz. Comme il apparaît clairement que le diamètre d'un ion polyatomique est supérieur à celui de l'analyte de masse identique, il en ressort que l'ion polyatomique subit un plus grand nombre de collisions lors de son parcours dans la zone de collision, lesquelles s'accompagnent d'une perte énergétique supérieure. Il devient donc possible de dissocier les deux populations d'ions. En effet, une barrière énergétique judicieusement appliquée permet de séparer les deux populations en bloquant les polyatomiques moins énergétiques. On parle dans ce cas d'une discrimination en énergie cinétique (DEC ou KED - Kinetic Energy Discrimination) $(16,17)$. Cette séparation est réalisée par l'augmentation du potentiel transversal entre le multipôle inséré dans la cellule de collision/réaction et le quadripôle du filtre de masse. Cette discrimination est d'autant plus efficace que l'énergie des ions est faible et bien contrôlée dans tous les degrés de liberté. On parle dans ce cas de thermalisation du faisceau ionique. Cette technique ne peut toutefois s'appliquer aux interférences isobariques pures, comme ${ }^{40} \mathrm{Ar}$ sur le ${ }^{40} \mathrm{Ca}$, par exemple. Dans ce cas, en effet, il convient de mettre en œuvre un processus plus réactionnel, tel que décrit ci-dessous.

\section{Les réactions de modification de l'interférent}

Divers types de réactions peuvent être exploités afin d'éliminer l'interférence polyatomique ou isobarique. De plus, certaines conditions doivent être remplies pour permettre la levée des interférences : la réaction se doit d'être thermodynamiquement possible, c'està-dire qu'elle soit exothermique ou suffisamment peu endothermique pour que la thermalisation incomplète des ions suffise à compenser le léger manque énergétique. Il convient également qu'elle soit cinétiquement utile, à savoir que la vitesse de réaction du gaz avec l'interférent soit suffisante pour permettre une interaction rapide. Enfin, il est nécessaire que cette réaction du gaz avec l'analyte soit suffisamment lente, s'il s'avère que celleci est également thermodynamiquement possible. Les gaz généralement utilisés dans ces cas sont l'hydrogène, l'ammoniac, le méthane... De nombreuses applications de ces procédés ont été développées dans tous les domaines analytiques (18) (tableau II).

\section{Réaction de condensation de l'analyte}

Dans certaines circonstances, en particulier en cas d'interférences multiples, une autre technique peut être utilisée qui consiste à faire réagir l'analyte avec un gaz réactif. L'ion moléculaire ainsi formé est alors analysé en tant que tel. Cette technique peut, par exemple, être illustrée par l'usage d' $\mathrm{O}_{2}$ comme gaz de réaction, pour le dosage du ${ }^{80} \mathrm{Se}$ dans des matrices riches en ${ }^{40} \mathrm{Ca}$,
Tableau II : Liste non-exhaustive d'interférences résolues par un gaz de collision ou de réaction, tous constructeurs et applications confondus.

\begin{tabular}{|c|c|c|c|}
\hline Analyte & $\begin{array}{l}\text { Abondance } \\
\text { isotopique } \\
\text { naturelle } \%\end{array}$ & Interférent & Gaz utilisés \\
\hline${ }^{23} \mathrm{Na}$ & 100 & $\mathrm{BC}$ & $\mathrm{H}_{2}$ \\
\hline${ }^{24} \mathrm{Mg}$ & 79,0 & $\mathrm{CC}$ & $\mathrm{H}_{2} / \mathrm{He}$ \\
\hline${ }^{27} \mathrm{AI}$ & 100 & $\mathrm{CN}$ & $\mathrm{He} / \mathrm{NH}_{3} / \mathrm{H}_{2}$ \\
\hline${ }^{28} \mathrm{Si}$ & 92,2 & $\mathrm{CO}, \mathrm{N}_{2}$ & $\mathrm{O}_{2} / \mathrm{NH}_{3} / \mathrm{H}_{2}$ \\
\hline${ }^{31} \mathrm{P}$ & 100 & $\mathrm{NO}$ & $\mathrm{Xe} / \mathrm{O}_{2}$ \\
\hline${ }^{32} \mathrm{~S}$ & 95,0 & $\mathrm{O}_{2}$ & $\mathrm{Xe} / \mathrm{O}_{2}$ \\
\hline${ }^{39} \mathrm{~K}$ & 93,3 & $\mathrm{ArH}$ & $\mathrm{H}_{2} / \mathrm{NH}_{3}$ \\
\hline${ }^{40} \mathrm{Ca}$ & 96,9 & Ar & $\mathrm{H}_{2} / \mathrm{NH}_{3} / \mathrm{CH}_{4}$ \\
\hline${ }^{51} \mathrm{~V}$ & 99,8 & $\mathrm{CIO}, \mathrm{CIN}$ & $\mathrm{He} / \mathrm{NH}_{3}$ \\
\hline${ }^{52} \mathrm{Cr}$ & 83,8 & ArC & $\mathrm{He} / \mathrm{H}_{2} / \mathrm{NH}_{3} / \mathrm{CH}_{4}$ \\
\hline${ }^{53} \mathrm{Cr}$ & 9,5 & $\mathrm{ArC}, \mathrm{CIO}$ & $\mathrm{NH}_{3}$ \\
\hline${ }^{54} \mathrm{Fe}$ & 5,8 & ArN & $\mathrm{He} / \mathrm{H}_{2} / \mathrm{NH}_{3} / \mathrm{CH}_{4}$ \\
\hline${ }^{55} \mathrm{Mn}$ & 100 & ArNH, CIO & $\mathrm{He} / \mathrm{H}_{2} / \mathrm{NH}_{3} / \mathrm{CH}_{4}$ \\
\hline${ }^{56} \mathrm{Fe}$ & 91,8 & $\mathrm{ArO}$ & $\begin{array}{c}\mathrm{He} / \mathrm{Xe} / \mathrm{H}_{2} / \mathrm{NH}_{3} \\
/ \mathrm{CH}_{4}\end{array}$ \\
\hline${ }^{57} \mathrm{Fe}$ & 2,1 & $\mathrm{ArOH}, \mathrm{CaOH}$ & $\mathrm{He}$ \\
\hline${ }^{58} \mathrm{Ni}$ & 68,1 & $\operatorname{ArO}(\mathrm{H})$ & $\mathrm{He} / \mathrm{H}_{2} / \mathrm{NH}_{3} / \mathrm{CH}_{4}$ \\
\hline${ }^{59} \mathrm{Co}$ & 100 & $\mathrm{ArOH}$ & $\mathrm{He} / \mathrm{H}_{2} / \mathrm{NH}_{3} / \mathrm{CH}_{4}$ \\
\hline${ }^{60} \mathrm{Ni}$ & 26,2 & $\mathrm{ArO}, \mathrm{CaO}$ & $\mathrm{He} / \mathrm{H}_{2} / \mathrm{NH}_{3} / \mathrm{CH}_{4}$ \\
\hline${ }^{63} \mathrm{Cu}$ & 69,1 & $\mathrm{ArNa}$ & $\mathrm{He} / \mathrm{H}_{2} / \mathrm{NH}_{3} / \mathrm{CH}_{4}$ \\
\hline${ }^{64} \mathrm{Zn}$ & 48,3 & $\mathrm{SO}_{2}, \mathrm{~S}_{2}$ & $\mathrm{He} / \mathrm{H}_{2}$ \\
\hline${ }^{65} \mathrm{Cu}$ & 30,9 & $\mathrm{SO}_{2} \mathrm{H}, \mathrm{S}_{2} \mathrm{H}, \mathrm{ArMg}$ & $\mathrm{He}$ \\
\hline${ }^{66} \mathrm{Zn}$ & 28,0 & $\mathrm{ArMg}, \mathrm{SO}_{2}, \mathrm{~S}_{2}$ & $\mathrm{He} / \mathrm{H}_{2}$ \\
\hline${ }^{68} \mathrm{Zn}$ & 19,0 & $\mathrm{SO}_{2}, \mathrm{~S}_{2}$ & $\mathrm{He}$ \\
\hline${ }^{72} \mathrm{Ge}$ & 27,3 & ArAr & $\mathrm{He} / \mathrm{H}_{2}$ \\
\hline${ }^{74} \mathrm{Se}$ & 36,7 & ArAr & $\mathrm{H}_{2} / \mathrm{CH}_{4}$ \\
\hline${ }^{75} \mathrm{As}$ & 100 & ArCI & $\begin{array}{c}\mathrm{He} / \mathrm{H}_{2} / \mathrm{NH}_{3} / \mathrm{O}_{2} \\
/ \mathrm{CH}_{4}\end{array}$ \\
\hline${ }^{76} \mathrm{Se}$ & 9,4 & $\mathrm{ArAr}$ & $\mathrm{H}_{2} / \mathrm{O}_{2} / \mathrm{CH}_{4}$ \\
\hline${ }^{77} \mathrm{Se}$ & 7,6 & ArCI & $\mathrm{He} / \mathrm{H}_{2} / \mathrm{D}_{2}$ \\
\hline${ }^{78} \mathrm{Se}$ & 23,8 & ArAr & $\mathrm{H}_{2} / \mathrm{O}_{2} / \mathrm{CH}_{4} / \mathrm{D}_{2}$ \\
\hline${ }^{80} \mathrm{Se}$ & 49,6 & ArAr, $\mathrm{HBr}$ & $\mathrm{H}_{2} / \mathrm{O}_{2} / \mathrm{CH}_{4}$ \\
\hline${ }^{82} \mathrm{Se}$ & 8,7 & ArHArH & $\mathrm{He} / \mathrm{H}_{2}$ \\
\hline${ }^{84} \mathrm{Sr}$ & 0,6 & ${ }^{86} \mathrm{Kr}$ & $\mathrm{CH}_{4}$ \\
\hline${ }^{86} \mathrm{Sr}$ & 9,9 & ${ }^{86} \mathrm{Kr}$ & $\mathrm{CH}_{4}$ \\
\hline${ }^{87} \mathrm{Sr}$ & 7,0 & ${ }^{87} \mathrm{Rb}$ & $\mathrm{CH}_{3} \mathrm{~F}$ \\
\hline${ }^{90} \mathrm{Sr}$ & Radioisotope & ${ }^{90} \mathrm{Y},{ }^{90} \mathrm{Zr}$ & $\mathrm{O}_{2}$ \\
\hline${ }^{90} \mathrm{Zr}$ & 51,5 & ${ }^{40} \mathrm{Ar}^{50} \mathrm{Cr}$ & $\mathrm{H}_{2}$ \\
\hline${ }^{93} \mathrm{Nb}$ & 100 & ${ }^{40} \mathrm{Ar}^{53} \mathrm{Cr}$ & $\mathrm{H}_{2}$ \\
\hline${ }^{109} \mathrm{Ag}$ & 48,2 & $\begin{array}{c}\mathrm{NbO}, \mathrm{ZrOH}, \\
\mathrm{MoOH}\end{array}$ & $\mathrm{O}_{2}$ \\
\hline${ }^{111} \mathrm{Cd}$ & 12,8 & $\begin{array}{c}\mathrm{MoO}, \mathrm{ZrOH}, \\
\mathrm{MoOH}\end{array}$ & $\mathrm{O}_{2} / \mathrm{He} / \mathrm{H}_{2}$ \\
\hline${ }^{129} \mathrm{I}$ & Radioisotope & ${ }^{129} \mathrm{Xe}$ & $\mathrm{O}_{2}$ \\
\hline
\end{tabular}


où, à l'interférence ${ }^{40} \mathrm{Ar}^{40} \mathrm{Ca}$ s'ajoute celle du dimère d'argon $\left({ }^{40} \mathrm{Ar}^{40} \mathrm{Ar}\right)$. Après réaction avec l'oxygène, le sélénium peut alors être analysé sous forme d'oxydes ${ }^{80} \mathrm{Se}^{16} \mathrm{O}$ (19). Une autre application de ce procédé est illustrée par l'analyse du soufre et du phosphore ; ces éléments étant en effet eux-mêmes fortement interférés par le dimère d'oxygène dans le cas $d^{3}{ }^{32} \mathrm{~S}$ et par l'ion moléculaire $\mathrm{NO}$ en ce qui concerne le ${ }^{31} \mathrm{P}$, ils sont alors transformés en oxyde par réaction avec de l'oxygène et analysés sous cette forme (20).

\section{Systèmes de filtration}

Si l'utilisation des dispositifs de collision/réaction se montre d'une réelle efficacité en ce qui concerne la suppression des interférences, on assiste cependant au cours des réactions qui se produisent dans le dispositif, à des réactions parasites qui induisent la formation d'ions susceptibles de devenir à leur tour des interférents. Il s'agit donc de sous-produits issus de réactions secondaires du fait d'impuretés présentes dans le gaz utilisé, d'une thermalisation incomplète permettant à l'énergie cinétique des ions de se transformer en énergie potentielle lors d'une réaction endothermique, ou de plusieurs réactions chimiques différentes se produisant simultanément dans la cellule. A titre d'exemple, la présence de traces de carbone dans le dispositif (issu de l'échantillon, des impuretés présentes dans le gaz, de relents d'huile issus de l'appareil...) peut générer toute une série de molécules carbonées susceptibles de devenir à leurs tours des interférents (18). En conséquence, les constructeurs se sont vus contraints d'éliminer ces interférences par l'utilisation d'un filtre. Deux types sont utilisés actuellement : le filtre énergétique et le filtre de masse.

\section{Le filtre énergétique}

La discrimination en énergie cinétique peut en effet être utilisée une nouvelle fois, ces ions, sous-produits de réactions secondaires, étant moins énergétiques que les ions d'analytes issus du plasma (21).

\section{Le filtre de masse}

On parle dans ce cas de résolution chimique ou de PICS (Post Ionization Chemical Separation). Le principe de ce filtre est basé sur une élimination sélective des ions constitutifs des interférents polyatomiques, lors de leur apparition, en fonction de leur masse. Une bande passante de stabilité en masse, permettant bien entendu le passage de la masse de l'analyte, est créée dans la cellule au moyen du quadripôle qui s'y trouve installé. Cette bande de stabilité est optimisée de manière synchrone avec la masse analysée par le spectromètre de masse (17). Compte tenu des plages de stabilité des ions dans les différents multipôles $(22,23)$, seul le quadripôle possède les caractéristiques nécessaires pour assurer cette filtration en masse.

\section{Conclusions sur les dispositifs de collision/ réaction}

Au cours des années qui ont suivi l'introduction des dispositifs de collision/réaction, les développements applicatifs réalisés par les différents constructeurs ont permis d'offrir aux analystes une potentialité importante de réduction des interférences pour un coût limité (tableau II). Seuls quelques cas particuliers subsistent qui se traduisent par des limites de détection encore élevées, comme pour l'analyse du silicium, par exemple, pour lequel l'interférence due à ${ }^{28} \mathrm{~N}_{2}$ s'est avérée peu sensible aux divers types de gaz utilisés. Les dispositifs de collision/réaction constituent donc un outil de mesure susceptible d'être utilisé en analyse de routine. Toutefois, le recul acquis depuis l'introduction des cellules voilà quelques années, a permis de mettre en évidence un certain nombre de limitations. En effet, au cours de mesures d'isotopie par exemple, on constate une influence du flux et du choix du gaz, ainsi que des réglages du filtre de masse ou énergétique, sur le biais de masse. Cette observation a été rapportée par plusieurs auteurs et notamment sur les rapports ${ }^{87} \mathrm{Sr} /{ }^{86} \mathrm{Sr}$ (24) et ${ }^{34} \mathrm{~S} /{ }^{32} \mathrm{~S}(25)$. Elle semble liée à une différence dans l'efficacité de transmission des ions de distributions énergétiques différentes.

On observe également l'apparition de certains effets de matrice induisant des sensibilités différentes en fonction de la matrice, comme par exemple lors de l'analyse du vanadium ou du chrome en matrice biologique (26). La présence relativement nouvelle sur le marché de l'interface de collision/réaction (CRI) ne nous permet pas encore de tirer de réelles conclusions quant à ces différentes interférences.

\section{Les toutes dernières modifications}

Outre les interférences spectrales, les ICP-MS souffrent cependant d'un autre handicap, à savoir leur tolérance limitée parrapport aux matrices chargées. Cette limitation provient essentiellement de l'interface constituée d'une paire de cônes portés à haute température et prompts à collecter nombre d'atomes constitutifs de la matrice, modifiant, par là même, la sensibilité de l'appareil. Cette limitation a été prise en compte par certains constructeurs qui ont adapté la forme des cônes, ainsi que pour l'un d'entre eux, la forme de certaines lentilles, au type d'analyse réalisé. Le choix pourra donc s'établir entre des cônes performants en terme de sensibilité mais exhibant une tolérance limitée vis-à-vis de la matrice, et des cônes plus robustes face à la matrice mais qui seront moins performants question sensibilité. Par 
ailleurs, il est maintenant possible sur presque tous les appareils d'effectuer une injection d'oxygène de quelques dizaines de millilitres dans le flux d'argon de nébulisation ; cette option supplémentaire s'avérant indispensable lors de l'analyse directe de solvants organiques afin d'éviter l'accumulation de carbone sur les cônes. Enfin, plusieurs appareils offrent désormais la possibilité de piloter un diluteur en ligne, de contrôler un générateur d'hydrures ou de vapeurs froides, de réaliser des préconcentrations, voire d'effectuer des analyses en ligne (Analyse dans laquelle une préparation d'échantillon, telle que celles citées précédemment, est effectuée automatiquement avant quantification des analytes). Outre ces aspects matériels, les constructeurs ont également continué à optimiser les logiciels en permettant, par exemple, l'optimisation automatique des paramètres du système, l'optimisation du temps et de la technique de rinçage, l'intégration des contrôles Qualité au regard des exigences des normes américaines de l'EPA (Environmental Protection Agency) 6020, 200.8 et 1638 .

\section{Les analyses de spéciation par ICP-MS}

Depuis près de 10 ans pour les plus précoces d'entre eux, les fabricants se sont également attaché à développer les capacités analytiques de leurs ICP-MS en terme d'analyse de spéciation. Outre l'interfaçage entre un système HPLC et un ICP-MS qui ne posent aucun problème particulier, deux interfaces GC-ICPMS sont actuellement commercialement disponibles, et une troisième est sur le point de l'être. Véritables lignes de transfert destinées à conduire les espèces séparées par le processus chromatographique réalisé dans le four, ces interfaces se doivent de maintenir une température élevée afin de conserver la volatilité des espèces tout en évitant une diffusion et un élargissement de pics. Hormis ce côté matériel, presque tous les constructeurs se sont également astreints à automatiser au maximum ce type d'analyses en permettant un pilotage du système chromatographique et de l'ICP-MS par un logiciel commun, et en asservissant le début des analyses par l'ICP-MS à la fin de l'injection. La possible utilisation d'un passeur automatique et des facilités dans le traitement des signaux transitoires finalisent le dispositif.

\section{Conclusion}

Sur l'ensemble des ICP-MS vendus actuellement dans le monde, les systèmes quadripolaires équipés d'un dispositif de collision/réaction en constituent la majorité. On estime en effet que les appareils équipés de ce type de système représentent $85 \%$ à $90 \%$ des ventes sur le marché mondial, et qu'il est fort probable qu'ils continueront à le dominer dans les années qui viennent. Toutefois les autres dispositifs rencontrent également la faveur d'un certain nombre d'utilisateurs en fonction de leurs applications, très spécifiques, et de leurs besoins.

\section{Remerciements}

J'adresse mes remerciements aux sociétés Agilent Technologies, GBC Scientific Equipment et à son distributeur Vinci, GV Instruments, Nu Instruments, PerkinElmer Instruments, Thermo Fisher Scientific et Varian, Inc. pour le prêt, l'accès aux ICP-MS, et/ ou le partage d'informations. J'exprime également ma gratitude à de nombreux membres du LCABIE et de UT2A pour les discussions techniques que j'ai pu avoir avec eux sur ce sujet.

\section{Références}

1. Allain P., Mauras Y., Douge C., Jaunault L., Delaporte T., Beaugrand C. Determination of iodine and bromine in plasma and urine by inductively coupled plasma mass spectrometry. Analyst 1990 ; 115 (6) : 813-15.

2. Allain P., Berre S., Premel-Cabic A., Mauras Y., Delaporte T. Concentrations of rare earth elements in plasma and urine of healthy subjects determined by inductively coupled plasma mass spectrometry. Clin. Chem. 1990 ; 36 (11) : 2011-12.

3. Allain P., Berre S., Premel-Babic A., Mauras Y., Delaporte T., Cournot A. Investigation of the direct determination of uranium in plasma and urine by inductively coupled plasma mass spectrometry. Anal. Chim. Acta 1991 ; 251 (1-2) : 183-5.

4. Wieser M.E., Schwieters J.B. The development of multiple collector mass spectrometry for isotope ratio measurements. Internat. J. Mass Spectrom. 2005 ; 242 : 97-115.

5. Rodushkin I., Bergman T., Douglas G., Engström E., Sörlin D., Baxter D.C. Authentication of Kalix (N.E. Sweden) vendace caviar using inductively coupled plasma-based analytical techniques: Evaluation of different approaches. Anal. Chim. Acta 2007; 583 (2) : 310-8.

6. Kylander M.E., Weiss D.J., Jeffries T.E., Kober B., Dolgopolova A., Garcia-Sanchez R., Coles B.J. A rapid and reliable method for $\mathrm{Pb}$ isotopic analysis of peat and lichens by laser ablation-quadrupole-inductively coupled plasma-mass spectrometry for biomonitoring and sample screening. Anal. Chim. Acta 2007; 582 (1) : 116-24.

7. Farkas J., Buhl D., Blenkinsop J., Veizer J. Evolution of the oceanic calcium cycle during the late Mesozoic : Evidence from _44/40Ca of marine skeletal carbonates. Earth Planet. Sci. Let. 2007 ; 253 (1-2) : 96-111. 
8. Balcerzak M. An overview of analytical applications of time of flight-mass spectrometric (TOF-MS) Analyzers and an inductively coupled plasma-TOF-MS technique. Anal. Scien. 2003 ; 19 : 979-89.

9. Infante H.G., Van Campenhout K., Blust R., Adams F.C. Anion-exchange high performance liquid chromatography hyphenated to inductively coupled plasma-isotope dilution-time-of-flight mass spectrometry for speciation analysis of metal complexes with metallothionein isoforms in gibel carp (Carassius auratus gibelio) exposed to environmental metal pollution. J. Chrom. A 2006 ; 1121 (2) : 184-90.

10. Scadding C.J., Watling R.J., Thomas A.G. The potential of using laser ablation inductively coupled plasma time of flight mass spectrometry (LA-ICP-TOF-MS) in the forensic analysis of micro debris. Talanta 2005 ; 67 (2) : 414-24.

11. Dimitrova-Koleva B., Benkhedda K., Ivanova E., Adams F. Determination of trace elements in natural waters by inductively coupled plasma time of flight mass spectrometry after flow injection preconcentration in a knotted reactor. Talanta 2007 ; 71 (1) : 44-50.

12. Paucot, H. Les dispositifs de collision/réaction en ICPMS : Revue descriptive et modes de fonctionnement. Spectra Analyse $2006 ; 252$ : 23-7.

13. Douglas D.J. Some current perspectives on ICP-MS. Can. J. Spectrosc. $1989 ; 34$ : 38-49.

14. Rowan J.T., Houk R.S. Attenuation of polyatomic ion interferences in inductively coupled plasma spectrometry by gas-phase collisions, Appl. Spectrosc. 1989 ; 43 : 976-80.

15. Turner P., Merren T., Speakman J. Interface studies in the ICP mass spectrometer, in plasma source mass spectrometry developments and applications, Grenville Holland and Scott D. Tanner, Eds, The Royal Society of Chemistry, $1997: 28-34$.

16. Yamada N., Takahashi J., Sakata K. The effects of cellgas impurities and kinetic energy discrimination in an octopole collision cell ICP-MS under non-thermalized conditions. J. Anal. At. Spectrom. 2002 ; 17 : 1213-22.
17. Hattendorf B., Gunther, D. Suppression of in-cell generated interferences in a reaction cell ICP-MS by bandpass tuning and kinetic energy discrimination. J. Anal. At. Spectrom. $2004 ; 19: 600-6$.

18. Tanner S., Baranov V., Bandura D. Reaction cells and collision cells for ICP-MS : a tutorial review. Spectrochim. Acta Part B 2002 ; 57 : 1361-452.

19. Hattendorf B., Gunther D. Strategies for method development for an inductively coupled mass spectrometer with bandpass reaction cell. Approaches with different reaction gases for the determination of selenium. Spectrochim. Acta B 2003 ; 58 : 1-13.

20. Bandura D., Baranov V., Tanner, S. Detection of ultratrace phosphorus and sulfur by quadrupole ICP-MS with dynamic reaction cell. Anal. Chem. 2002 ; 74 : 14971502.

21. Du Z., Houk R.S. Attenuation of metal oxide ions in inductively coupled plasma mass spectrometry with hydrogen in a hexapole collision cell. J. Anal. At. Spectrom. $2000 ; 15: 383-8$.

22. Hagg C., Szabo I. New ion-optical devices utilizing oscillatory electric fields. II. Stability of ion motion in a two-dimentional hexapole field. Int. J. Mass Spectrom. Ion Process. 1986 ; 73 : 237-75.

23. Hagg C., Szabo I. New ion-optical devices utilizing oscillatory electric fields. III. Stability of ion motion in a two-dimentional octapole field. Int. J. Mass Spectrom. Ion Process. 1986 ; 73 : 277-94.

24. Vanhaecke F., Balcaen L., Deconinck I., Mass discrimination in dynamic reaction cell (DRC)-ICP-Mass Spectrometry. J. Anal. At. Spectrom. 2003 ; 18 : 1060-5.

25. Masson P., Kaspers K., Van Bergen M. Determination of sulfur isotope ratios and concentrations in water samples using ICP-MS incorporating hexapole ion optics. J. Anal. At. Spectrom. 1999 ; 14: 1067-74.

26. Bonnefoy C., Menudier A., Moesch C. Determination of Chromium in Whole Blood by DRC-ICP-MS : Spectral and Non-spectral Interferences, Anal. Bioanal. Chem. $2005 ; 383$ : 167-73. 